\title{
The Development of Support for People Following COVID-19 Infection in Kyrgyzstan: Survey Study of Patients and Clinical Workers
}

\section{Aijan Taalaibekova ( $\square$ aijan_taalaibekova@bk.ru )}

national center of cardiology and internal medicine https://orcid.org/0000-0001-8439-4710

Alena Oleinik

national center of cardiology and internal medicine

Kamila Magdieva

National Center of Cardiology and Internal Medicine

Gulzada Mirzalieva

National Center of Cardiology and Internal Medicine

Zainab K. Yusuf

University of Leicester Department of Respiratory Sciences

Maamed Mademilov

national center of cardiology and internal medicine

\section{Azamat Akylbekov}

national center of cardiology and internal medicine

\section{Amy V. Jones}

Loughborough University School of Sport Exercise and Health Sciences

Ruhme B. Miah

University of Leicester Department of Respiratory Sciences

Dominic Malcolm

Loughborough University School of Sport Exercise and Health Sciences

\section{Andy Barton}

University Hospitals Plymouth NHS Trust

\section{Talant Sooronbaev}

national center of cardiology and internal medicine

\section{Sally J. Singh}

University of Leicester Department of Respiratory Sciences

Mark W. Orme

University of Leicester Department of Respiratory Sciences 
Keywords: COVID-19, Survey, Rehabilitation, SARS-CoV-2, post-COVID support.

Posted Date: November 11th, 2021

DOI: https://doi.org/10.21203/rs.3.rs-1010492/v1

License: (c) (1) This work is licensed under a Creative Commons Attribution 4.0 International License. Read Full License 


\section{Abstract}

Background: After the acute period from SARS-CoV-2 infection, many COVID-19 survivors continue to have ongoing symptoms. There is a need to render assistance to such COVID-19 survivors in Kyrgyzstan but it is unclear how support should be designed. This study aimed to examine what this support should look like, specifically the persisting post-COVID symptoms, views on the optimal timing, suitable delivery methods, content of the support, and obstacles to implementation.

Methods: This study was conducted by using two cross-sectional online surveys in the Russian language. The first group included clinical workers of various specialties who worked in the places of treatment for patients with COVID-19. The second group consisted of patients aged $\geq 18$ years who had recovered from COVID-19 who were recruited from family medicine centers using the database of COVID-19 survivors. The survey was conducted anonymously and voluntarily, and consisted of 16 questions.

Results: 85 clinical workers and 132 COVID-19 survivors took part in the survey. COVID-19 survivors reported they would be willing to devote 1-3 sessions per a week (84\%) with a duration of no more than 1 hour $(90 \%)$ to post-COVID interventions. Respondents identified the Internet (43\%) and separate smartphone applications (42\%) as the best media via which to receive support at home. The greatest barrier to accepting help was the risk of re-infection (43\%). According to clinical workers, the main goal of post-COVID-19 support should be the amelioration of breathing problems $(70 \%)$, increased physical exercise $(57 \%)$ and elements of psychological support (57\%). Clinical workers reported that support should begin during treatment for COVID-19 (65\%), or immediately after returning from the hospital (59\%). Respondents stated that it would be better to carry out support at home via video conference $(65 \%)$, or by means of telephone calls $(47 \%)$. The cost of treatment $(47 \%)$ and the individual's employment at work (43\%) were deemed potential barriers to COVID-19 survivors participating in support interventions.

Conclusions: Clinical workers and COVID-19 survivors deemed post-COVID-19 support necessary. Our results will directly inform the development and delivery of post-COVID support interventions in Kyrgyzstan and Central Asia, in combination with the views and needs identified directly from people with persisting post-COVID symptoms.

\section{Background}

At the end of 2019, in the Chinese province of Hubei, in the city of Wuhan, a new coronavirus was identified, severe acute respiratory syndrome coronavirus-2 (SARS-CoV-2), which caused the development of a respiratory infection epidemic throughout China. This was followed by an increase in the number of cases worldwide [1]. As of October 9th 2021, according to John Hopkins University, the number of cases of infection worldwide reached almost 233 million people, the number of deaths was about 4.7 million and is growing every day [2]. The pandemic has led to numerous problems for healthcare systems [3], with almost every country experiencing disruptions in the provision of health services, with the greatest 
difficulties faced by low- and middle-income countries (LMICs) [4]. For many patients, there are longerlasting symptoms persisting well beyond the acute and sub-acute phases of infection, termed post-COVID syndrome [5].

In Kyrgyzstan, the pandemic has caused considerable disruption and burden to individuals, society and the economy. According to the Ministry of Health of Kyrgyzstan, as of October 9th 2021, there have been more than 127,000 cases and more than 2,600 deaths [6]. In clinical practice, doctors often encounter post-COVID syndrome [7] and patients report long-lasting symptoms that reduce their quality of life (QoL). In particular, some survivors cannot go to work and others find it difficult to carry out their daily activities. In Kyrgyzstan, as with other Central Asia countries, little is known about individuals with post-COVID syndrome and this has been acknowledged as a research priority by the Ministry of Health.

For many LMICs, there is no existing infrastructure or services to support the rehabilitation of COVID-19 survivors, as has been suggested in other parts of the world [7, 8]. For example, most high-income countries have adapted existing rehabilitation services, which already support a wide range of health conditions through multidisciplinary teams $[9,10]$. This has been particularly apparent for symptomatic patients [12] who share similar symptoms (e.g. breathlessness, cough and fatigue) to people attending Pulmonary Rehabilitation (PR) [13]. In contrast, PR is not widely available in LMICs [13, 14], including Kyrgyzstan. Additional post-COVID symptoms such as post-traumatic stress disorder (PTSD) and coping with social isolation must also be considered, but the extent to which individuals in Kyrgyzstan are burdened by the wide range of symptoms documented in other parts of the world [16-19] is unclear. Efforts to develop rehabilitation for COVID-19 survivors have been successful in some low resource settings [20] but there is a clear need to develop interventions specific to the context in which they will be delivered.

With the lack of existing rehabilitation infrastructure in Kyrgyzstan, the development of appropriate support for the many COVID-19 survivors with post-COVID syndrome is needed. This study aimed to examine what this support should look like, specifically address persisting post-COVID symptoms, views on the optimal timing, suitable delivery methods, content of the support, and obstacles to implementation. To do this, we conducted online surveys with COVID-19 survivors and clinical workers in Kyrgyzstan.

\section{Methods}

\section{Study design}

This study was conducted using two cross-sectional online surveys in Russian. The survey was designed by the National Institute for Health Research (NIHR) Global RECHARGE Group for Pulmonary Rehabilitation (17/63/20). Ethical approval was obtained from the University of Leicester (\#24736). The study is reported in accordance with The Strengthening the Reporting of Observational Studies (STROBE) guidelines [21]. 


\section{Participants and setting}

The first group included clinical workers of various specialties (including pulmonologists, infectious disease specialists, and nurses) from various medical institutions (National Center for Cardiology and Internal Medicine [NCCIM], National Hospital, City Clinical Hospital \#6, Family Medicine Center \#7, National Center for Maternity and Childhood Protection, Republican Infectious Clinical Hospital) who worked in the places of treatment for patients with COVID-19.

The second group consisted of patients aged $\geq 18$ years who had recovered from COVID-19 (with or without confirmation of Polymerase Chain Reaction (PCR), with varying degrees of severity), who were recruited from family medicine centers using a database of COVID-19 survivors. Patients had received an online consultation from a pulmonologist, visited an outpatient clinic or were hospitalized at the NCCIM, in Bishkek, Kyrgyzstan.

\section{Variables and data sources}

The survey was conducted anonymously and voluntarily, and consisted of 16 questions. The survey for clinical workers included questions on the most important considerations that should be part of postCOVID-19 support, the best timing and location of support, and barriers to referring patients to support. The patient survey included questions on the problems they faced due to SARS-CoV-2 infection, the best way to receive support, what time commitment they would give to support their recovery, and barriers to participating in post-COVID-19 support. The respondents were provided with a link to the survey and the questions were filled out on devices (tablet, phone, and computer) independently, or with the help of a researcher, if required.

\section{Statistical methods}

Data were described using frequencies and percentages, with no inferential statistics due to the descriptive nature of the surveys. Data were summarized using reports from Jisc Online Surveys ${ }^{\circledR}$ and Microsoft Excel ${ }^{\circledR}$. Qualitative data counting was used to analyse the free text responses, which involved identifying similar responses within the data and grouping them together [22].

\section{Results}

\section{Survey completion and participant characteristics}

For COVID-19 survivors, 132 were invited to participate, of which 111 (84\%) completed the survey (68\% female, $51 \%$ aged $19-29$ years). The majority of SARS-CoV-2 survivors surveyed were in paid employment (41\%), in full-time education (20\%) or retired (10\%). Most (77\%) were diagnosed as a result of contacting medical institutions with the help of clinical workers, with laboratory confirmation. Two-thirds selfisolated following diagnosis, $50 \%$ were prescribed medications, $18 \%$ were admitted to hospital and $2 \%$ were admitted to Intensive Care Unit. 
For clinical workers, 87 were invited to take part in the survey, of which $85(98 \%)$ completed the survey (84\% female, $53 \%$ aged $19-29$ years). The main group of surveyed clinical workers consisted of pulmonologists (25\%), nurses (12\%), infectious disease specialists $(9 \%)$ and general practitioners of modern medicine (9\%). The majority of clinical workers were actively involved in working with COVID-19 survivors: $42 \%$ were engaged in their treatment in a hospital, $21 \%$ were engaged in making a diagnosis, $20 \%$ worked on an outpatient basis, $12 \%$ delivered oxygen therapy, whilst $28 \%$ had not worked directly with COVID-19 patients.

\section{Responses of clinical workers}

The survey asked clinical workers to list their five most important topics to support COVID-19 survivors. The top five selected topics were 'advice on managing breathlessness' (66\%), 'advice on medications' (58\%), 'aerobic exercise/regaining fitness levels' (45\%), 'support for anxiety or depression' (45\%), and 'advice on managing fatigue' (31\%) (Figure 1). The five least selected items were 'support for social isolation' (8\%), 'financial advice' (7\%), 'learning how to find trustworthy information' (4\%), 'resistance exercise/strength training' (1\%), and 'support for coping with stigma' (1\%).

When asked whether support should be provided to patients who were not hospitalized with SARS-CoV-2, $92 \%$ of clinical workers stated that support should be given. When asked where support should be delivered, most clinical workers selected community settings (55\%) or at home (55\%), with $37 \%$ suggesting hospitals. The majority of clinical workers were confident that patients would be receptive of support with their post-COVID recovery $(88 \%)$. Some (9\%) were unsure how patients would respond to the offer of support.

Barriers to the provision and acceptance of support according to clinical workers can be seen in Figure 2 . According to clinical workers, the main barriers to providing support to patients were cost of treatment (47\%) and patient employment/financial concerns (43\%). Inadequate supply of PPE kits for healthcare professionals (39\%) was also of great importance. Factors clinical workers believed would hinder patient acceptance of support were belief in the value of non-medical treatments (46\%), cost of treatment (41\%) and patient employment/financial concerns (39\%).

Most clinical workers reported that support be provided to COVID-19 survivors either during treatment $(65 \%)$ or immediately after returning home from the hospital (59\%). Clinical workers selected video consultation as the most suitable method of communication to deliver support (65\%), followed by telephone calls (47\%), dedicated smartphone application (45\%) and the use of television (45\%). Almost a third of clinical workers (31\%) selected face-to-face communications or home visits.

Overall, clinical workers were positive about supporting the recovery of COVID-19 survivors, with $73 \%$ reporting they think it would be beneficial for patients.

\section{Responses of COVID-19 survivors}


Problems faced by patients as a result of COVID-19 and the areas in which they wanted help can be seen in Figure 3. The most common were tiredness or fatigue (67\%), loss of physical strength (51\%), loss of smell (51\%), coughing (43\%) and loss of taste (41\%).

When patients were asked, 'How would you like to receive such support and how would you like to receive such assistance?', the most common approaches were receiving support at home with digital support $(43 \%)$, at home with telephone support (33\%), or receiving a home visit from a clinical worker (29\%), as seen in Figure 4.

When patients were asked 'How much time per day and how many days per week they are willing to devote to participating in an intervention to alleviate their symptoms after COVID-19', they responded as ready to devote $1-3$ sessions per a week ( $84 \%)$ with a duration of no more than 1 hour $(90 \%)$.

Factors that could prevent patients from accepting rehabilitation assistance after COVID-19 can be seen in Figure 5. Risk of re-infection was the main barrier for patients accepting support (43\%), followed by inconsistent information about COVID-19 (27\%) and lack of confidence in healthcare professionals (23\%). Almost a quarter (24\%) of patients reported not needing additional support.

\section{Discussion}

The data were obtained from patients who experienced COVID-19 and various clinical workers who dealt with COVID-19 survivors in Kyrgyzstan. Based on our results, a post-COVID support intervention could be home-based, for hospitalized and non-hospitalized patients, comprising 1-3 sessions/week lasting up to 1 hour, with a combination of face-to-face, video and telephone support; with a focus on strength and aerobic training, and managing symptoms of fatigue, cough, loss of taste and smell, breathlessness, anxiety and depression. There is a need to address patient barriers to accepting support which includes risk of re-infection, inconsistent information about COVID, a lack of confidence in clinical workers and financial concerns. Barriers to clinical workers referring patients for such support included inadequate supplies of PPE.

\section{Structure and timing of the support}

The importance of rehabilitation after COVID-19 has been emphasized according to the framework of the International Classification of Functioning, Disability and Health [23]. Taking into account the opinion of the surveyed clinical workers, the developed rehabilitation program requires an integrated approach, including exercise as a major component in supporting COVID-19 survivors. It should be noted that the majority of the respondents also noted the importance of psychological support. Isolated mental health issues or psychological issues, such as post-traumatic stress disorder, anxiety, and depression are documented in SARS-CoV-2 infection [24]. The Respiratory Rehabilitation Committee of the Chinese Association of Rehabilitation Medicine, and Cardiopulmonary Rehabilitation Group of the Chinese Society of Physical Medicine and Rehabilitation suggest that, apart from easing respiratory distress, improving physical function and QoL, rehabilitation programs can be useful in improving both anxiety and 
depression [24]. Six-week respiratory rehabilitation can improve respiratory function, and anxiety in elderly patients with COVID-19, but it has no significant improvement in elderly depressive state and activities of daily living [25]. In addition, particular attention should be paid to the fight against shortness of breath and fatigue, which have been consistently identified as some of the most common symptoms [26]. Rehabilitation assessment should be offered to all patients suffering from post-COVID-19 symptoms, not just laboratory-confirmed patients or only those that have been hospitalized [27].

Regarding the issue of the location of rehabilitation, most COVID-19 survivors and clinical workers consider community settings and home as the most suitable places. This may be associated with a perceived reduced risk of (re)infection and/or community transmission, compared with attending hospitals. Moreover, one should take into account the fact that most of the Kyrgyz population use public transport for daily travel, which increases the risk of infection. Still, one third of the respondents preferred the inpatient facility to be more suitable for rehabilitation, possibly due to the availability of professional medical care.

According to clinical workers, a complex range of support measures for COVID-19 survivors should begin during treatment for COVID-19, or immediately after discharge from the hospital. This may shorten the recovery time. There is evidence of a positive impact on early mobilization of SARS-CoV-2 survivors [28]. Several studies have reported the beneficial effects of hospital exercise programs on patients with pneumonia. For example, an inpatient rehabilitation intervention (daily 50-minute sessions for 8 days, including stretching, resistance and walking exercises) improved functional ability, muscle strength, QoL, and shortness of breath [29].

It should be noted that COVID-19 survivors were of the same opinion and would like to receive home rehabilitation via the Internet and telephone, suggesting that they want to avoid unnecessary contact with others and minimize the risk of re-infection. The world already has data on the experience of remote rehabilitation. For example, a study from Japan used video and audio communications from an iPad to provide remote support [30]. No serious adverse events were observed. Remote rehabilitation was an effective and safe method against transmission and could facilitate the rehabilitation of patients in COVID-19 wards [27]. Digital methods are gaining popularity in Kyrgyzstan every year. Their advantages are availability, ease of handling and elimination of the risk of infection. With modified communications, remote rehabilitation has significant potential use in a wide variety of situations in the near future, including those at high risk of infection or those that can be treated at a distance, such as at home or in remote locations [27]. At normal times, we use face-to-face meetings which has been the primary method of communicating with patients. But, during the pandemic, consultations by telephone have been very successful [31]. However, it should be noted that this is a very simple method and one cannot see the patient or show any exercises. Consequently, nearly a third of clinical workers and COVID-19 survivors opted for in-person or home visits, possibly because older adults and clinical workers are reluctant to use the Internet. In addition, in many remote villages of Kyrgyzstan there is no Internet or mobile communication. 
When COVID-19 survivors were asked about the frequency of the sessions, the most suitable schedule would be 1-3 days a week with the duration of the session not more than 1 hour. Patients noted some barriers that would prevent them from accepting support. The risk of re-infection was the main obstacle for accepting support, in line with preference on location of support, and followed by conflicting information about COVID-19 and mistrust of clinical workers. This may be due to the fact that at the beginning of the COVID-19 pandemic it was unfamiliar to the population, and the procedures for the diagnosis and treatment of this disease were not known, and therefore there were mortality rates. In contrast to the opinion of clinical workers, almost a quarter of COVID-19 survivors reported that they did not need additional support.

\section{Main issues/symptoms to address}

The majority of COVID-19 survivors noted the importance of rehabilitation to eliminate persistent symptoms, restore lost or reduced functions of various organs and systems, and for the subsequent improvement of QoL. In our study, the main long-term symptoms for COVID-19 survivors were tiredness or fatigue, loss of physical strength, cough, and loss of smell and taste. A systematic review has shown that symptoms of COVID-19 usually persist after the acute phase of infection, with implications for healthrelated functioning and QoL [32]. There is now a large amount of data on the long-term symptoms experienced by COVID-19 survivors after being discharged from the hospital. An Italian study that followed 143 patients 7 weeks after discharge found that $53 \%$ of patients reported fatigue, which is similar to our data, $43 \%$ reported shortness of breath, $27 \%$ reported joint pain and $22 \%$ reported chest pain [15]. In a similar study in the UK, fatigue associated with a new illness was the most common symptom, reported by $72 \%$ of participants in the intensive care group and $60.3 \%$ of participants in the ward group. The next common symptoms were shortness of breath $(65.6 \%$ in the intensive care group and $42.6 \%$ in the ward group) and psychological stress (46.9\% in the intensive care group and $23.5 \%$ in the ward group) [18]. Judging by the data from our review and from other countries, we can say that the main and leading symptom is fatigue and shortness of breath.

\section{Barriers to referral and to participation}

Clinical workers and COVID-19 survivors highlighted common barriers in providing and receiving support of COVID-19 survivors care, such as the cost of treatment, the impact on patient employment and financial problems. Differences were also found; for example, the necessity of PPE kits for clinical workers. In addition, for COVID-19 survivors receiving rehabilitation, one of the main obstacles was the credibility of non-medical therapies. The lack of specialists and doubts about the quality of the prescribed drugs influence residents of Kyrgyzstan to use non-medical therapies. For example, methods such as bloodletting, drinking tinctures from plants, and eating certain foods (e.g., garlic, soda, onions) are gaining popularity, with traditional medicines highly popular in many LMICs.

\section{Limitations of the study}


This exploratory study recruited a convenience sample and data collection were limited to an online survey, which may have led to selection bias. We attempted to minimise selection bias by supporting patients without their own device to complete the survey. Due to limitations in staffing and resources, we were able to recruit a small proportion of patients hospitalised with COVID-19 and the study may have benefited from in-depth, qualitative exploration. However, the inclusion of free-text responses was sufficient to clearly identify barriers to setting up post-COVID-19 support interventions. The results of this study provide a valuable initial insight into how to develop and implement post-COVID support in Kyrgyzstan, which may also facilitate wider intervention development in Central Asia, but monitoring of the implementation will be required.

\section{Conclusions}

COVID-19 survivors and clinical workers are receptive to the provision of support for ongoing symptoms. However, the views of clinical workers and patients did not always align. Our findings will be used to help design and deliver appropriate post-COVID-19 support activities for people living in Kyrgyzstan and Central Asia. Based on our findings, we propose the following initial structure for such support: a homebased intervention, comprising 2-3 sessions/week lasting up to 1 hour, delivered primarily through video and telephone communication, and with a focus on strength and aerobic training and managing symptoms of fatigue, cough, loss of taste and smell, breathlessness, anxiety and depression. The main patient barriers to accepting support include the risk of re-infection, inconsistent information about COVID-19, a lack of confidence in clinical workers and financial concerns. Barriers to clinical workers referring patients for such support included cost of treatment, patient employment status and inadequate supplies of PPE.

\section{Abbreviations}

COVID-19

Corona Virus Disease 19

SARS-Cov-2

Severe Acute Respiratory Syndrome Corona virus 2

\section{LMICs}

Low- and Middle-Income Countries

QoL

Quality of life

PR

Pulmonary Rehabilitation

PTSD

Post-Traumatic Stress Disorder

NIHR

National Institute for Health Research 
NCCIM

National Center for Cardiology and Internal Medicine

PCR

Polymerase Chain Reaction

PPE

Personal protective equipment

UK

United Kingdom

\section{Declarations}

\section{Ethics approval and consent to participate}

Ethical approval was obtained from the University of Leicester (\#24736).

\section{Availability of data and materials}

The datasets of the current study are available from the corresponding author upon reasonable request.

\section{Competing interests}

There are no competing interests.

\section{Funding}

This project is funded by the National Institute for Health Research (NIHR) Global RECHARGE (17/63/20) and the University of Leicester International Research Development Funding Call (M631PC27). The views expressed are those of the author(s) and not necessarily those of the NIHR or the Department of Health and Social Care.

\section{Authors' contributions}

AT, AO, KM, GM, ZKY, MM, AA, MWO, AVJ, RM, DM, TS, and SJS participated in concept and design of the survey. $A T, A O, K M, G M, Z K Y, M M, A A$, and MWO participated in data analysis and interpretation of results. AT drafted a manuscript, which was then reviewed by ZKY, MWO, DM, AB, AVJ, TS, and SJS. All authors contributed intellectually to this paper, read and approved the final version for submission for peer review and publication.

\section{Acknowledgements}

We gratefully acknowledge the all participants for taking part in this survey.

\section{References}


[1] Q. Ye, B. Wang, M. S. Jianhua, M. Junfen, S. Shang, and M. S. Qiang, “Epidemiological analysis of COVID - 19 and practical experience from China," no. January, pp. 1-15, 2020, doi: 10.1002/jmv.25813.

[2] "COVID-19 Dashboard by the Center for Systems Science and Engineering (CSSE) at Johns Hopkins University (JHU)." .

[3] "WHO Director-General's opening remarks at the media briefing on COVID-19 - 11 March 2020." https://www.who.int/director-general/speeches/detail/who-director-general-s-opening-remarks-at-themedia-briefing-on-covid-19--11-march-2020.

[4] "In WHO global pulse survey, $90 \%$ of countries report disruptions to essential health services since COVID-19 pandemic." https://www.who.int/news/item/31-08-2020-in-who-global-pulse-survey-90-ofcountries-report-disruptions-to-essential-health-services-since-covid-19-pandemic.

[5] National Institute for Health and Care Excellence, R. C. of G. Practitioners, and H. I. Scotland, "COVID19 rapid guideline: managing the long-term effects of COVID-19," NICE Guidel., no. 18 December 2020, pp. 1-35, 2020.

[6] "Ministry of Health of the Kyrgyz Republic." http://med.kg/index.php/en/.

[7] "NICE COVID-19 rapid guidelines," PharmacoEconomics Outcomes News, vol. 877, no. 1, pp. 33-33, 2021, doi: 10.1007/s40274-021-7682-3.

[8] S. J. Singh et al., "British Thoracic Society survey of rehabilitation to support recovery of the postCOVID- 19 population," BMJ Open, vol. 10, no. 12, pp. 1-10, 2020, doi: 10.1136/bmjopen-2020-040213.

[9] I. Grigoletto, V. Cavalheri, F. F. de Lima, and E. M. C. Ramos, "Recovery after COVID-19: The potential role of pulmonary rehabilitation," Brazilian J. Phys. Ther., vol. 24, no. 6, pp. 463-464, 2020, doi: 10.1016/j.bjpt.2020.07.002.

[10] E. Crisafulli et al., "Efficacy of standard rehabilitation in COPD outpatients with comorbidities," Eur. Respir. J., vol. 36, no. 5, pp. 1042-1048, 2010, doi: 10.1183/09031936.00203809.

[11] L. E. G. W. Vanfleteren et al., "Clusters of comorbidities based on validated objective measurements and systemic inflammation in patients with chronic obstructive pulmonary disease," Am. J. Respir. Crit. Care Med., vol. 187, no. 7, pp. 728-735, 2013, doi: 10.1164/rccm.201209-16650C.

[12] T. Greenhalgh, M. Knight, C. A'Court, M. Buxton, and L. Husain, "Management of post-acute covid-19 in primary care," $B M J$, vol. 370, 2020, doi: 10.1136/bmj.m3026.

[13] E. Daynes, C. Gerlis, S. Briggs-Price, P. Jones, and S. J. Singh, "COPD assessment test for the evaluation of COVID-19 symptoms," Thorax, vol. 76, no. 2, pp. 185-187, 2021, doi: 10.1136/thoraxjnl2020-215916. 
[14] G. M. Habib et al., "Systematic review of clinical effectiveness, components, and delivery of pulmonary rehabilitation in low-resource settings," npj Prim. Care Respir. Med., vol. 30, no. 1, 2020, doi: 10.1038/s41533-020-00210-y.

[15] F. M. Bickton, C. Fombe, E. Chisati, and J. Rylance, "Evidence for pulmonary rehabilitation in chronic respiratory diseases in sub-Saharan Africa: A systematic review," Int. J. Tuberc. Lung Dis., vol. 24, no. 10, pp. 991-999, 2020, doi: 10.5588/IJTLD.19.0526.

[16] L. F. F. the G. against C.-19 post-acute care study group. Carfi A, Bernabei R, "Persistent symptoms in patients after acute COVID-19.," JAMA., vol. 324(6):, 2020.

[17] K. Ramanathan et al., "the COVID-19 resource centre - inc," no. January, pp. 19-21, 2020.

[18] B. Davido, S. Seang, R. Tubiana, and P. De Truchis, "Since January 2020 Elsevier has created a COVID-19 resource centre with free information in English and Mandarin on the novel coronavirus COVID19. The COVID-19 resource centre is hosted on Elsevier Connect, the company 's public news and information ," no. January, 2020.

[19] S. J. Halpin et al., "Postdischarge symptoms and rehabilitation needs in survivors of COVID-19 infection: A cross-sectional evaluation," J. Med. Virol., vol. 93, no. 2, pp. 1013-1022, 2021, doi: 10.1002/jmv.26368.

[20] F. M. Bickton, E. Chisati, J. Rylance, and B. Morton, "An Improvised Pulmonary Telerehabilitation Program for Postacute COVID-19 Patients Would Be Feasible and Acceptable in a Low-Resource Setting," Am. J. Phys. Med. Rehabil., vol. 100, no. 3, pp. 209-212, 2021, doi: 10.1097/PHM.0000000000001666.

[21] E. von Elm, D. G. Altman, M. Egger, S. J. Pocock, P. C. Gøtzsche, and J. P. Vandenbroucke, “The strengthening the reporting of observational studies in epidemiology (STROBE) statement: Guidelines for reporting observational studies," Int. J. Surg., vol. 12, no. 12, pp. 1495-1499, 2014, doi: 10.1016/j.ijsu.2014.07.013.

[22] L. B. Hannah DR, "Counting in Qualitative Research: Why to Conduct it, When to Avoid it, and When to Closet it2011;20(1):14-22. doi:10.1177/1056492610375988," J. Manag. Inq., pp. 2011;20(1):14-22. doi:10.1177/1056492610375988, [Online]. Available: https://doi.org/10.1177/1056492610375988.

[23] L. M. Sheehy, "Considerations for Postacute Rehabilitation for Survivors of COVID-19.," JMIR public Heal. Surveill., vol. 6, no. 2, p. e19462, May 2020, doi: 10.2196/19462.

[24] A. B. Siddiq, F. A. Rathore, D. Clegg, and J. J. Rasker, "Pulmonary Rehabilitation in COVID-19 patients: A scoping review of current practice and its application during the pandemic," vol. 66, no. 4, pp. 480-494, 2020, doi: 10.5606/tftrd.2020.6889.

[25] K. Liu, W. Zhang, Y. Yang, J. Zhang, Y. Li, and Y. Chen, “Since January 2020 Elsevier has created a COVID-19 resource centre with free information in English and Mandarin on the novel coronavirus COVID- 
19. The COVID-19 resource centre is hosted on Elsevier Connect, the company 's public news and information ," no. January, 2020.

[26] D. L. Sykes, L. Holdsworth, N. Jawad, P. Gunasekera, A. H. Morice, and M. G. Crooks, "Post-COVID-19 Symptom Burden: What is Long-COVID and How Should We Manage It?," Lung, vol. 199, no. 2, pp. 113119, 2021, doi: 10.1007/s00408-021-00423-z.

[27] 0. O'Sullivan et al., "Rehabilitation post-COVID-19: cross-sectional observations using the Stanford Hall remote assessment tool.," BMJ Mil. Heal., 2021, doi: 10.1136/bmjmilitary-2021-001856.

[28] B. Puchner et al., "Beneficial effects of multi-disciplinary rehabilitation in postacute COVID-19: An observational cohort study," Eur. J. Phys. Rehabil. Med., vol. 57, no. 2, pp. 189-198, 2021, doi: 10.23736/S1973-9087.21.06549-7.

[29] et. al C. Stephensa, "of the original source. These permissions are granted for free by Elsevier for as long as the COVID-19 resource centre remains active .," Impacts COVID-19 Agric. food Syst. Worldw. Prog. to Sustain. Dev. goals, no. January, pp. 1-3, 2020.

[30] T. SAKAI, C. HOSHINO, R. YAMAGUCHI, M. HIRAO, R. NAKAHARA, and A. OKAWA, "Remote rehabilitation for patients with covid-19," J. Rehabil. Med., vol. 52, no. 9, 2020, doi: 10.2340/165019772731.

[31] J. Car, G. C. H. Koh, P. S. Foong, and C. J. Wang, "Video consultations in primary and specialist care during the covid-19 pandemic and beyond," BMJ, vol. 371, pp. 1-6, 2020, doi: 10.1136/bmj.m3945.

[32] T. Nasserie, M. Hittle, and S. N. Goodman, "Assessment of the Frequency and Variety of Persistent Symptoms among Patients with COVID-19: A Systematic Review," JAMA Netw. Open, vol. 4, no. 5, pp. 119, 2021, doi: 10.1001/jamanetworkopen.2021.11417.

\section{Figures}


Advice on managing breathlessness

Advice on medications

Aerobic exercise/regaining fitness levels

Support for anxiety or depression

Advice on managing fatigue

Advice on nutrition

Advice on home remedies from alternative medicines

Support for Post-Traumatic Stress Disorder (PTSD)

Infection control relating to COVID-19

Advice on managing cough

Dealing with grief

Lung function testing

Integrating back into the community

Support for managing other health conditions

Monitoring symptoms

Advice on returning to work

Support for social isolation

Financial advice

Learning how to find trustworthy information

Resistance exercise/strength training

Support for coping with stigma

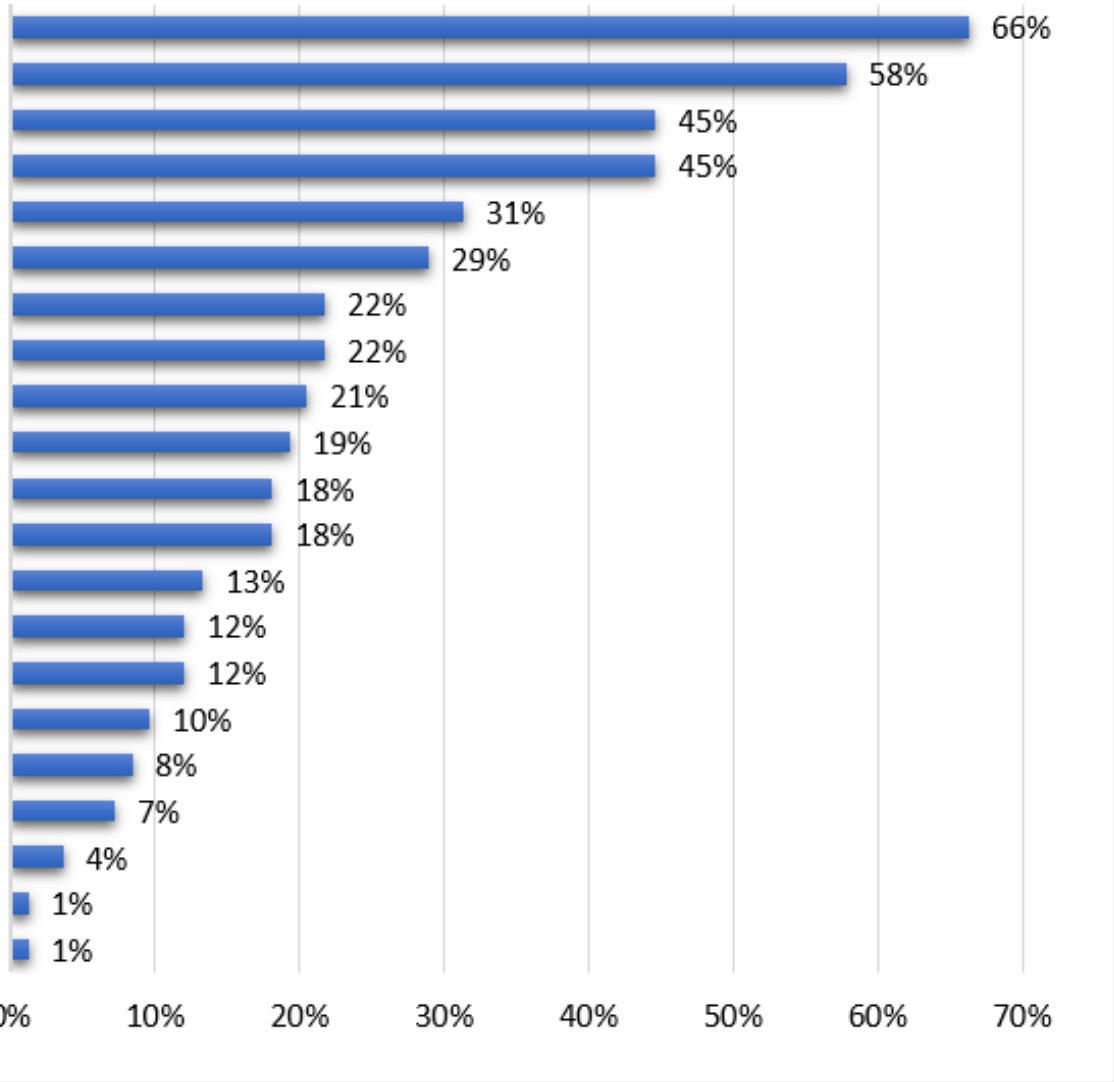

\section{Figure 1}

Which of the following potential considerations should be part of post-COVID-19 support? 
Patient employment/financial concerns

Inadequate supply of PPE kits for healthcare professionals

Belief in the value of non-medical treatments

Risk of patient spreading infection in the community

Risk of patient being re-infected

Risk of patients infecting healthcare staff

Lack of social support for the patient

Uncertainty of non-medical treatments

Stigma experienced by patients

Time commitment for healthcare professionals

No perceived need for support

Transport needs of patient

Patient's condition too poor to participate

Lack of time to make referrals

Time commitment for patient

Patient's condition too good to participate

Risk of patients exercising

Patient living alone

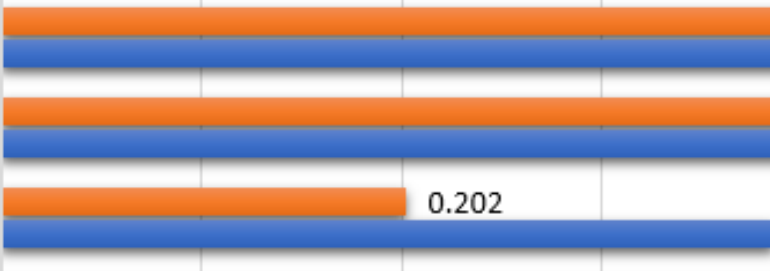

0.405
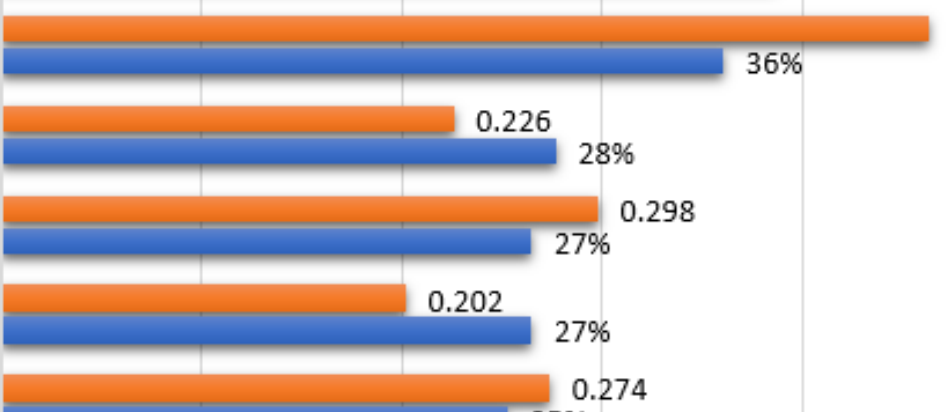

0.298
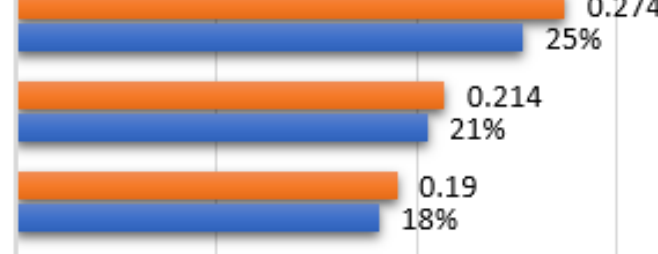

0.214

$21 \%$
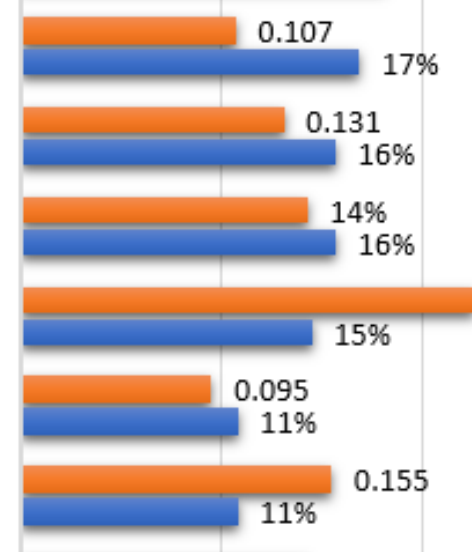

0.226

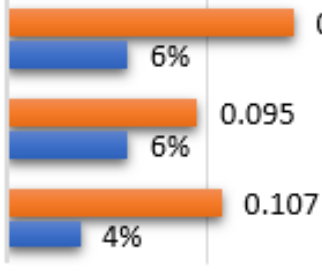

0.143

$0 \%$

$10 \%$

$20 \%$

$30 \%$

$40 \%$

a barriers to patients accepting a referral to post COVID-19 support according to clinical workers

barriers to clinical workers referring patients

\section{Figure 2}

Which of the following may be barriers to clinical workers referring patients to post COVID-19 support and what may be barriers to patients accepting a referral to post COVID-19 support according to clinical workers? 


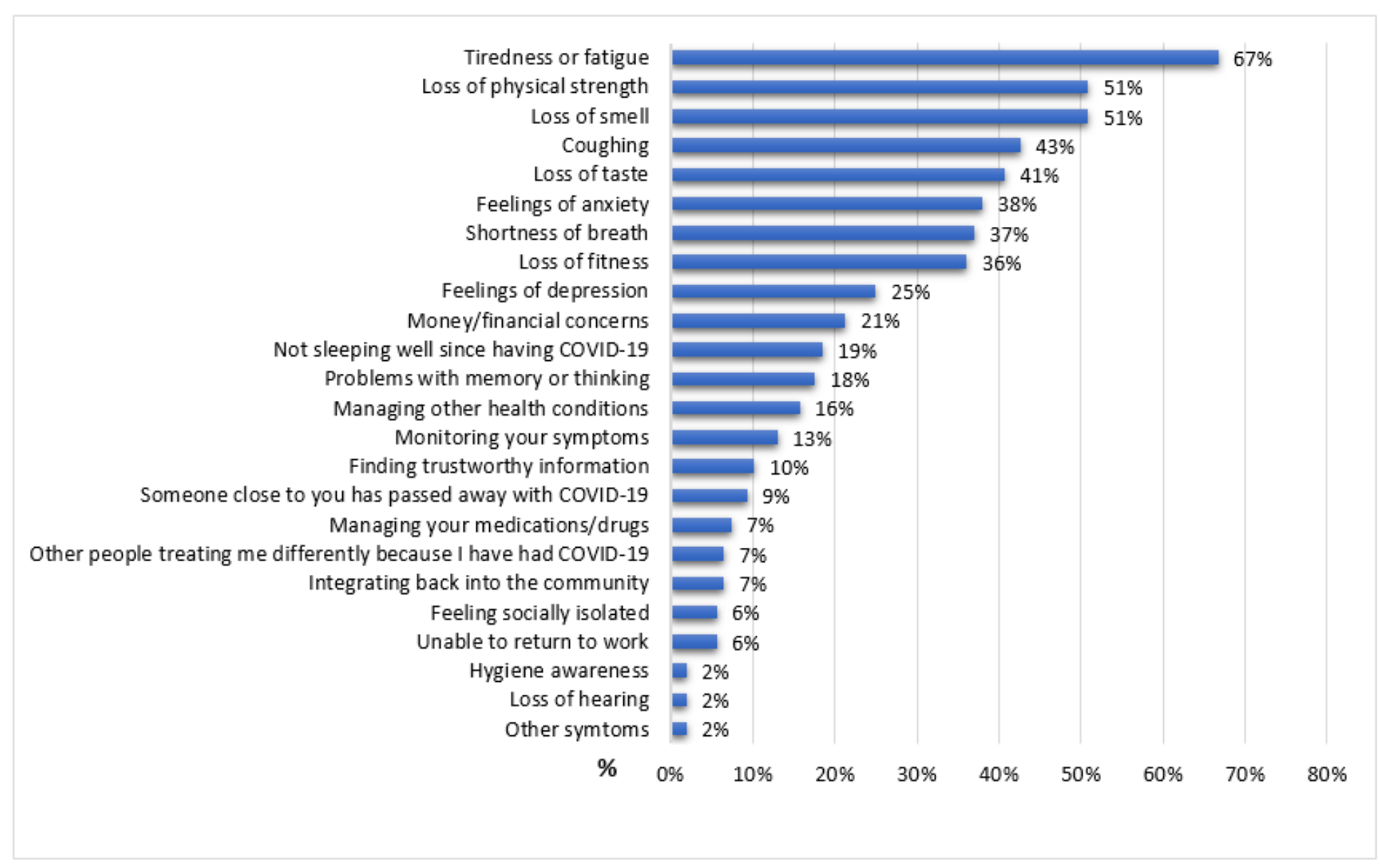

\section{Figure 3}

What problems are you facing as a result of COVID-19 and what would you want help with?

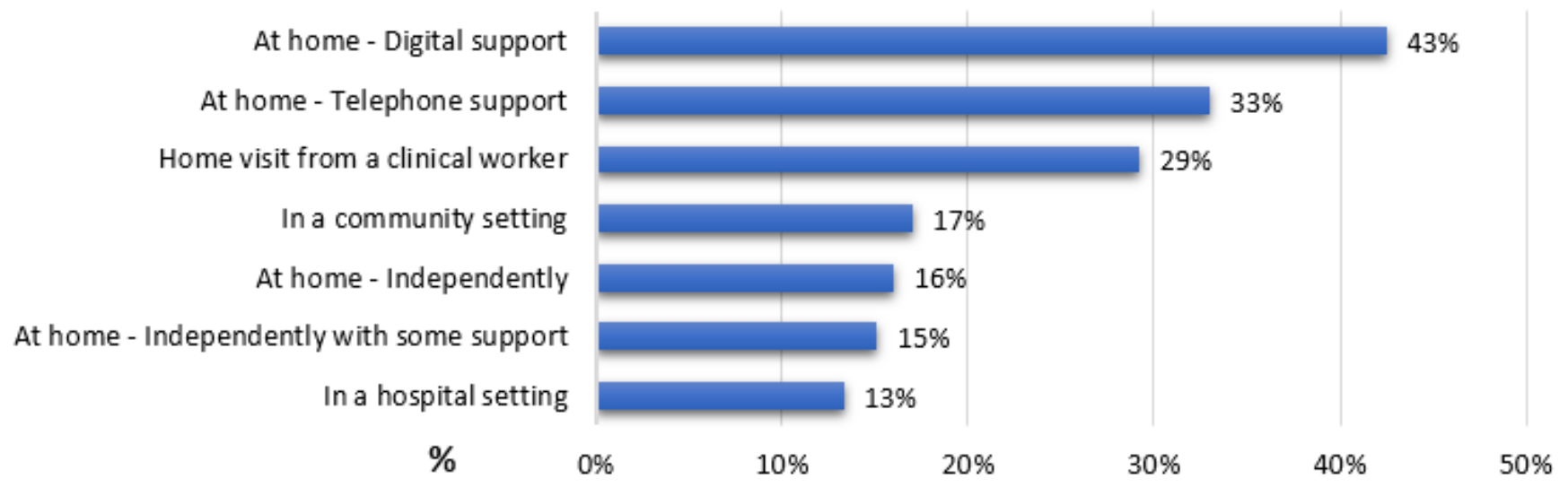

\section{Figure 4}

How would you like to receive such support and how would you like to receive such assistance? 
Risk of re-infection

Existence of inconsistent information about COVID-19

Employment/financial concerns

Do not need any additional support

Lack of confidence in healthcare professionals

Time commitment for myself

Feeling anxious or depressed

The availability of vaccines or drugs for COVID-19

Costs

Risk of infecting healthcare professionals

The value of non-medical treatments

Lack of social support

Risk of exercising

Risk of spreading infection in their community

Family would prefer me to rest

Time commitment for my family

Transport needs

Pre-existing health conditions

Prejudice or discrimination from other people

Previous exercise experience

Living alone

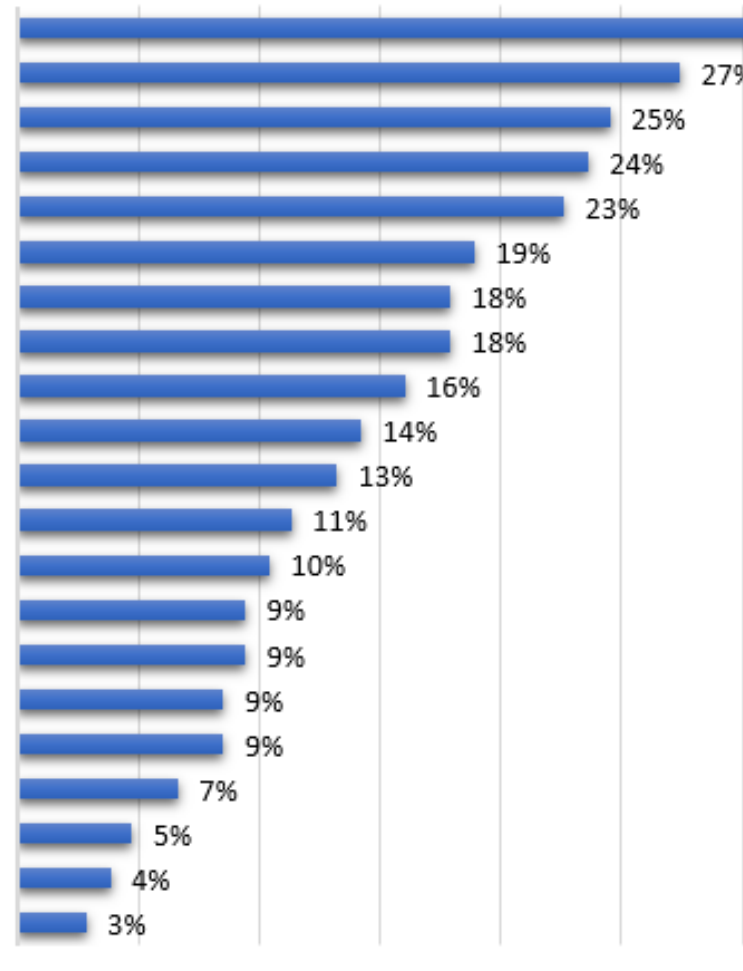

$\% \quad 0 \%$

\section{Figure 5}

What might make it difficult for you to access support to help you recover from COVID-19? 